\title{
Radial Line Slot Antenna Design for Monopulse Space Debris Radar
}

\author{
M. Sierra Castañer , J.I. Gayá Fuertes, J.M. Seguí Gómez de Olea , R. Martín Gallego , J.L. Fernández Jambrina
}

\begin{abstract}
This paper shows the design of a radial line slot antenna for Space Debris Radar Applications. The monopulse antenna has two beams, sum and difference, and requires an amplitude and phase receiver for detection of the azimuth and elevation direction of arrival. The antenna is circular polarized, and this paper focus on the design of the receiver antenna. The paper shows the optimization process of the position and length of slots in the central frequency of $94 \mathrm{GHz}$ and a first analysis of the feeding network. This design will be tested with the fabrication of a prototype
\end{abstract}

Index Terms - radial line slot antenna, monopulse, antenna synthesis, radar, space debris.

\section{INTRODUCTION}

Space Debris detection is becoming a challenge due to the increasing number of out of order satellites and other elements in orbit around the earth. According with European Space Agency [1], small objects (from 1 to $10 \mathrm{~cm}$ of diameter), are the most potentially harmful since they are too small to detect individually. Technical University of Madrid, together with other Universities works in a research project with the purpose of the study of Space Debris Radar at millimeter wave frequencies. Different monopulse antenna designs are analyzed in the project. This paper deals with the design aspects of a radial line slot antenna for this application. Previous studies were presented in [2]. In that paper, the idea of the monopulse antenna was implemented at $\mathrm{Ku}$ band. However, there was not an optimization process of the slot (position and length). This paper shows the optimization process for the slot arrangement and a study of the efficiencies obtained with this design depending on the number of rings of slots. The results will be checked with a prototype at a lower frequency.

The paper is divided in the following sections. Section II shows the specifications of the monopulse antenna and radar system. Section III shows the optimization process of the slots positions and lengths, Section IV shows the calculation of the antenna efficiencies and parameters with respect the radius, Section V the feeding structure, Section VI the validation and Section VII the conclusions.

The authors want to acknowledge the Madrid Region Governmen project for financing the project Space Debris Radar (S2013/ICE-3000 SPADERADAR-CM) and the Spanish Government, for the support of the project ENABLING5G "Enabling Innovative Radio Technologies for $5 \mathrm{G}$ networks" (TEC2014-55735-C3-1-R)

\section{ANTENNA SPECIFICATIONS}

The antenna is left hand circularly polarized. The gain of the final design is $35 \mathrm{dBi}$ at the central frequency of $94 \mathrm{GHz}$.
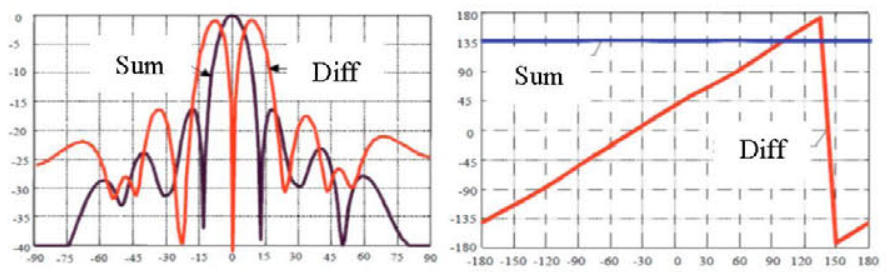

Fig. 1: Sum and difference amplitude and phase patterns

For this design the specified frequency band is very narrow, and only the analysis of the central frequency is required. The antenna is printed on a PTFE substrate: dielectric constant of 2.17 and width of $0.508 \mathrm{~mm}$. The monopulse antenna has two patterns: sum and difference (Fig. 1). For the detection of theta and phi angles, it is necessary to use amplitude and phase RADAR. Elevation is determined comparing the amplitude of both patterns (left side of Fig. 1), while azimuth is determined through the comparison of the phase of both patterns (right side of Fig. 1).

The required gain is $40 \mathrm{dBi}$ for this design, and the antenna is designed to maximize the gain at the central frequency. Both patterns are obtained with different excitations of the radial line slot antenna (RLSA). Basically, if the slots are placed in concentric rings, when the RLSA is excited in the centre, a conical or difference beam is generated. However, if a rotating mode is excited, a pencil or sum beam is generated. This rotating mode can be generated in different ways. The easier one is with 4 posts excited with $90 \mathrm{deg}$ of phase difference respect the previous one.

\section{OPTIMIZATION PROCESS OF SLOTS}

The antenna consists of circularly arranged rings of slots. In order to generate the sum pattern (pencil beam) a radial wave with a phase linearly dependent of the angular position is required. If the phase of the radial wave is constant with the angular position, a conical beam (difference pattern) is generated. 
The optimization algorithm is based on two algorithms: first a global algorithm based on simulated annealing is used and second a local one based on a conjugate gradient is used [3]. In this way, the number of iterations of the first algorithm is reduced.

Since the antenna is large enough, the RLSA is not finished in short circuit or absorber. On the other hand, the substrate is just extended. In order to assure that antenna radiates most of the power, the parameter to maximize is the difference between the directivity and the spillover power for the sum pattern (power after the last ring of slots). This optimization generates a uniform amplitude and phase excitation for the slots, assuring that most of the power is radiated through the slots. If the spillover efficiency were not included in the optimization function, the algorithm would do very small slots.

Also, as it is shown in [4], if the losses are not very high, the assumption of no losses in the radial line gives similar results to the case of lossy substrate. The analysis is performed using a Method of Moment algorithm with only 1 base function per slot and analytic expressions for the selfimpedance of slots and feed pins [5]. This makes possible to optimize large antennas in a reasonable time.

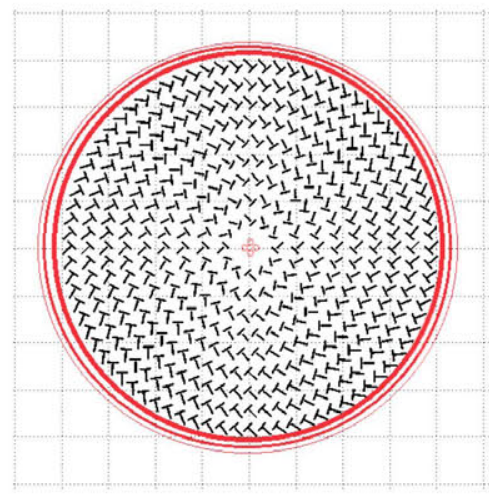

Fig. 2: Arrangement of slots after the optimization process for a 9-ring antenna.

\section{ANTENNA PARAMETERS AND EFFICIENCIES}

This optimization process has been applied to different sizes of antennas in order to analyze the gain of the antenna. The different antenna efficiencies are calculated in the following way.

- Aperture efficiency: the ratio between the calculated directivity and the maximum. This result is given after the end of the optimization process

- Spillover efficiency: the calculated through the residual power at the end of the antenna. This result is given after the end of the optimization process.

- Fabrication errors efficiency: a random error of $25 \mu \mathrm{m}$ in the length of each slot is introduced after the optimization process for each size of antenna. The result is compared with optimized antenna.

- Radiation efficiency: the connector losses are estimated in $1 \mathrm{~dB}$ and the losses in the radial line slot antenna are calculated as Eq. 1 [6].
$\operatorname{Loss}(\mathrm{dB})=0.6 \mathrm{R}(\mathrm{mm}) \alpha(\mathrm{dB} / \mathrm{mm})$

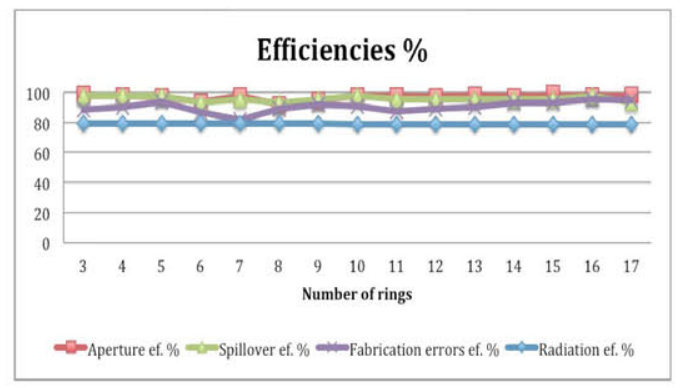

Fig. 3: Calculated efficiencies at central frequency depending of the number of rings.

The radiation efficiency is linear since it is calculated analytically. The other efficiencies are not linear since they are the results of the optimization process (aperture and spillover) or a random error in the length (fabrication errors efficiency). In any case, they are very high values. The antenna gain is calculated through the different efficiencies. It is observed that a very large antenna is required for getting 35 $\mathrm{dBi}$ (16 rings of slots). $40 \mathrm{dBi}$ would be obtained with 32 rings of slots. This value is calculated extrapolating the efficiencies shown if Fig. 3.

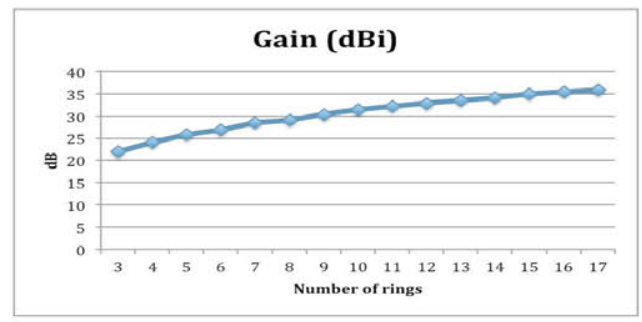

Fig. 4: Gain at central frequency depending of the number of rings.

\section{FEEDING STRUCTURE}

Different feeding structures have been studied for this proposed antenna. Both modes (for pencil and conical beams) are excited with different configuration of pins. The conical beam is excited through a pin located in the antenna center. For the conical beam, there are different possibilities to get the rotating mode. The ones presented in [2] and [7] were explored. The proposed solution is based in the structure of [2] (Fig. 5). In this case, there were four posts excited through a Butler Matrix. This Butler Matrix generated a sum pattern, when the four posts were excited with 90deg of phase difference and a difference pattern if the four slots were excited in phase. A central post in that design assured a good reflection coefficient for both pots. However, at this high frequency the design has to be simpler. The difference pattern has to be directly excited through a central pin and a feeding circuit based on waveguides is pre-designed for the sum pattern. Fig. 6 shows the placement of the 5 excitation pins in the RLSA 


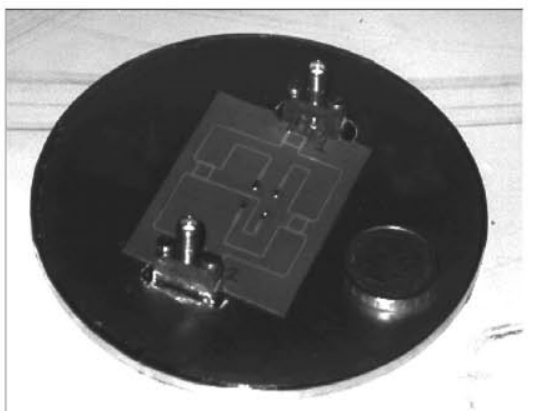

Fig. 5: Feeding structure designed in [2].

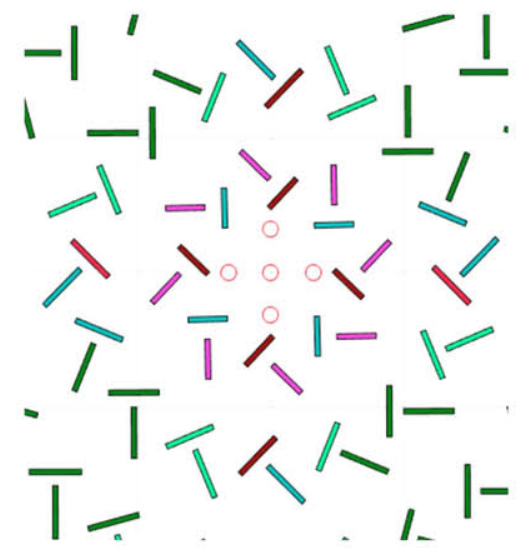

Fig. 6: 5 pins excitation for the proposed antenna.

A key aspect in the design is the design of these five pins. An optimization algorithm has been performed to design the length of the central post, the length of the side posts and the radial position of the side posts. The algorithm optimizes the reflection coefficient for the central post and one of the side posts (symmetrical situation). The isolation between both excitations is directly compensated due to the difference phase excitation of the four posts. The position and length of these posts are critical in the performance of the antenna.

\section{VALIDATION}

A 12 rings prototype at $24 \mathrm{GHz}$ has been designed and it is under construction. This antenna has been designed on a PTFE substrate. The measurements will be performed at least for the difference port (excited in the center with one coaxial) to check the validity of the design antenna.

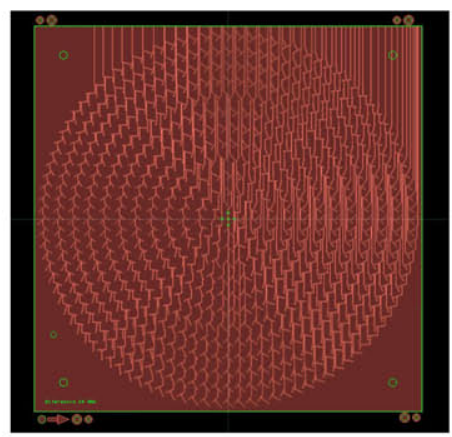

Fig. 7: Mask of the fabricated prototype
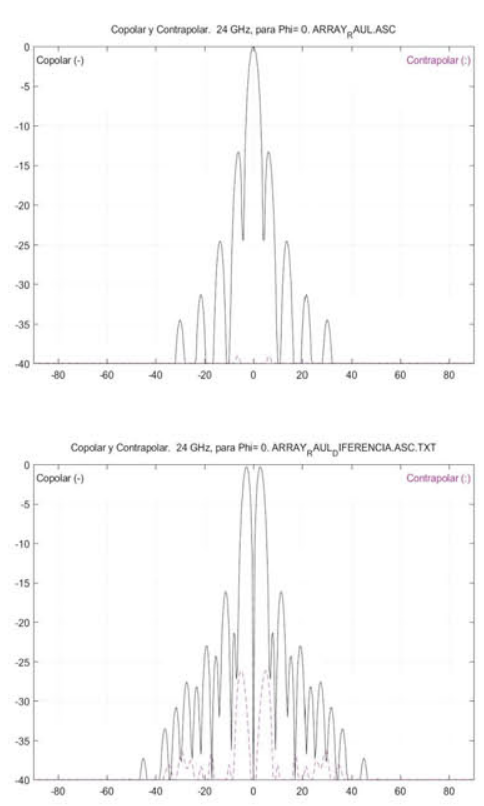

Fig. 8: Electromagnetic Simulation [5] for sum and difference patterns for the prototype ( $24 \mathrm{GHz}$ band)

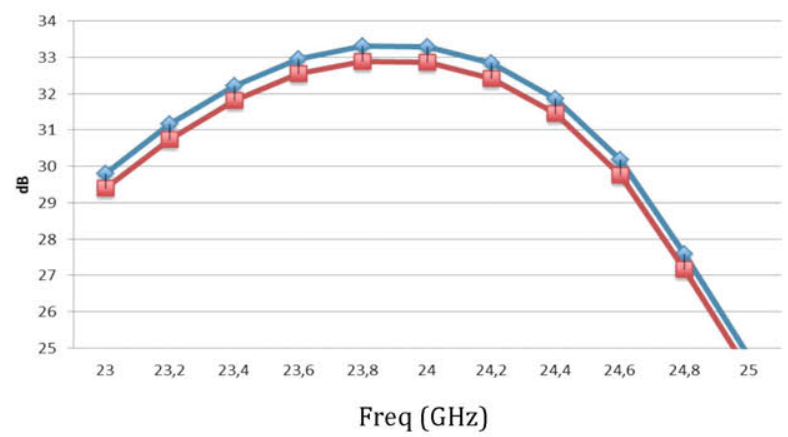

Fig. 9: Electromagnetic Simulation [5] for gain (red) and directivity (blue)

Fig. 8 shows the simulated sum and difference pattern at central frequency. Fig. 9 shows the expected gain and directivity between 23 and $25 \mathrm{GHz}$. It is observed that the antenna is narrow band as it was expected. It presents good performance in the band from 23.5 to $24.5 \mathrm{GHz}$, where the gain reduction with respect the peak is less than $1 \mathrm{~dB}$. In this band, the radiation patterns remains good enough.

\section{CONCLUSION}

This paper presents the design process of a monopulse radial line slot antenna at $94 \mathrm{GHz}$ for space debris detection. The paper has included the optimization process, the calculation of the antenna gain through different efficiencies. Finally, a feeding network based on $4+1$ pins is selected. The central pin generates the difference beam, while the four external pins generate the sum pattern, which will be excited through a waveguide (including power dividers and phase shifters). A first prototype of the difference pattern at $24 \mathrm{GHz}$ has been fabricated to validate the optimization process. 


\section{REFERENCES}

[1] ESA (European Space Agency), "El Efecto ESA. Al servicio de la cooperación y la innovación en Europa", 2014

[2] M. Sierra-Castañer, M. Sierra-Pérez, M. Vera-Isasa and J. L. FernándezJambrina, "Low cost monopulse Radial Line Slot Antenna", IEEE Trans. Antennas Propagat., vol. 51, pp. 256-263, 2003.

[3] T. Salmerón-Ruiz, T. Díez-Ricondo, M. Sierra-Castañer "An optimization procedure for Radial Line Slot Antennas with arbitrary pattern". Proceedings of 8th European Conference on Antennas and Propagation, EuCAP 2014. Den Haag (Netherlands). April 2014.

[4] T. Nguyen, J. Hirokawa, Makoto Ando, M. Sierra Castañer, "Design of mm-Wave RLSAs with Lossy Waveguides by Slot Coupling Control Techniques. IEICE Trans. Commu., Vol.E98-B, No.09,Sep. 2015.
[5] M. Sierra-Castañer, M. Sierra-Pérez, M. Vera-Isasa, J.L: Fernández Jambrina. "Fast analysis model for Radial Line Slot Antennas". Microwave and Optical Technology Letters (John Wiley \& Sons). Vol. 44, No.1, pp. 17-21. January 2005.

[6] T. Nguyen, R. Jayawardene, K. Sakurai, J. Hirokawa, M.Ando, M. Sierra Castaner, O. Amano, S. Koreeda, T. Matsuzaki, Y. Kamata "Propagation Characteristics of Honeycomb Structures Used in $\mathrm{mm}-$ Wave Radial Line Slot Antennas" IEICE Trans. Commu., Vol.E97-B, No.6 June 2014, pp.1139-1147.

[7] S. Hosono, J. Hirokawa, M. Ando, N. Goto, y H.Arai, "A rotating mode radial line slot antenna fed by a cavity resonator", IECE Trans. Commun., vol.E78-B, no.3, pp.407-413, March 1995. 